\title{
ARTICLE OPEN \\ Magneto-ionic control of spin polarization in multiferroic tunnel junctions
}

\author{
Yingfen Wei ${ }^{1}{ }^{1}$, Sylvia Matzen ${ }^{2 *}$, Cynthia P. Quinteros ${ }^{1}$, Thomas Maroutian ${ }^{2}$, Guillaume Agnus ${ }^{2}$, Philippe Lecoeur ${ }^{2}$ and $^{2}$ \\ Beatriz Noheda (iD) ${ }^{1,3 *}$
}

Multiferroic tunnel junctions (MFTJs) with $\mathrm{Hf}_{0.5} \mathrm{Zr}_{0.5} \mathrm{O}_{2}$ barriers are reported to show both tunneling magnetoresistance effect (TMR) and tunneling electroresistance effect (TER), displaying four resistance states by magnetic and electric field switching. Here we show that, under electric field cycling of large enough magnitude, the TER can reach values as large as $10^{6} \%$. Moreover, concomitant with this TER enhancement, the devices develop electrical control of spin polarization, with sign reversal of the TMR effect. Currently, this intermediate state exists for a limited number of cycles and understanding the origin of these phenomena is key to improve its stability. The experiments presented here point to the magneto-ionic effect as the origin of the large TER and strong magneto-electric coupling, showing that ferroelectric polarization switching of the tunnel barrier is not the main contribution.

npj Quantum Materials (2019)4:62; https://doi.org/10.1038/s41535-019-0201-0

\section{INTRODUCTION}

Combining the TMR effect (resistance change induced by magnetic field switching) of magnetic tunnel junctions (MTJs) with additional functionalities provided by the tunnel barrier, i.e., using multiferroic ${ }^{1}$ or ferroelectric ${ }^{2,3}$ layers as barriers, has drawn considerable attention driven by their potential application in multilevel memories. In these devices, four resistance states are achieved by means of both the TMR and the TER (resistance change by electric switching) effects. ${ }^{4-6}$ In addition, by combining two ferroic orders (ferromagnetic and ferroelectric), the coupling between the magnetic and electric degrees of freedom could realize electric field controlled spintronics, promising for the development of low-power and fast devices. ${ }^{6-12}$

The magnetoelectric (ME) coupling in heterostructures can have different origins. For instance, in artificial multiferroics made of ferroelectric and ferromagnetic layers, the ME coupling can be either strain-mediated or charge-mediated. ${ }^{13}$ The displacement of atoms under applied electric field in the ferroelectric barrier can affect the interface magnetization due to changes in hybridization. In addition, the magnetization of the ferromagnet, which is elastically coupled to the ferroelectric, can also change upon application of electric field due to magnetoelastic coupling. ${ }^{14-16}$ Alternatively, accumulation of spin-polarized carriers at the interface upon polarization of the dielectric ${ }^{17-19}$ can also give rise to $M E$ coupling. This effect is enhanced in the case of a ferroelectric, as a larger number of carriers will typically be necessary for screening. ${ }^{20-23}$ More recently, the magneto-ionic effect $^{24}$ has been proposed, by which the applied electric field induces ion migration, modifying the interfaces of the heterostructures and the properties of the layers.

In this work, tunnel barriers of crystalline $\mathrm{Hf}_{0.5} \mathrm{Zr}_{0.5} \mathrm{O}_{2}(\mathrm{HZO})$ are used in MTJs. Crystalline HZO grown under certain conditions has shown nanoscale ferroelectricity. ${ }^{25,26}$ Epitaxial growth of crystalline $\mathrm{HZO}$ can also be achieved ${ }^{27}$ and has been recently also demonstrated on perovskite substrates with $\mathrm{La}_{0.7} \mathrm{Sr}_{0.3} \mathrm{MnO}_{3}$
(LSMO) as bottom elecrode. ${ }^{28-30}$ The large band gap and high resistance of the $\mathrm{HZO}$ layer allows to fabricate full devices with extended electrodes for wire bonding, despite the low thickness of the barrier. This is not possible with perovskite ferroelectric (FE) tunnel barriers with such small thickness and, thus, so far these devices have been limited to investigation by scanning probes. ${ }^{31}$ Four resistance states have been obtained in this type of junctions by both magnetic and electric field switching, but no ME coupling was reported. ${ }^{31}$ Here we show that electric field cycling of high enough amplitude induces irreversible changes in the junction, which evolves from a negligible ME coupling state into a large ME coupling state. In the latter, sign reversal of the TMR effect is achieved by electrical switching, reversibly. Concomitantly, with increasing number of cycles, the TER increases to values up to $10^{6} \%$. In the following we discuss the mechanisms that lead to such phenomena.

\section{RESULTS}

Electrical switching of spin polarization

Multiferroic tunnel junctions (MFTJs) have been fabricated by integrating $2 \mathrm{~nm} \mathrm{HZO}$ tunnel barriers between top Co and bottom LSMO ferromagnetic (FM) electrodes. The HZO is highly crystalline and epitaxially grown on the LSMO electrode, which is in turn epitaxially grown on 001-oriented $\mathrm{SrTiO}_{3}$ (STO) substrates. As reported in ref. ${ }^{31}$, Trasmission Electron microscopy (TEM) demonstrates sharp interfaces with a roughness of the HZO film of $\sim 0.2 \mathrm{~nm}$. The schematic drawing of the devices used in the present work is shown in Fig. 1a. (See details in methods section).

By the electrical pulse switching protocol shown in Fig. 1b, the junction switches between the high resistance state, HRS $\left(R_{H}\right.$, after $\mathrm{V}+$ pulse) and the low resistance state, LRS ( $R_{L}$, after V-pulse). A voltage pulse with amplitude as large as $6 \mathrm{~V}$ is used in order to obtain the maximum resistance contrast (TER 400\%) (see Fig. 1c and ref. ${ }^{31}$ ). This resistance change is consistent with the change in

\footnotetext{
${ }^{1}$ Zernike Institute for Advanced Materials, University of Groningen, 9747 AG Groningen, The Netherlands. ${ }^{2}$ Centre for Nanoscience and Nanotechnology, CNRS UMR 9001, Université Paris-Sud, Université Paris-Saclay, 91120 Palaiseau, France. ${ }^{3}$ CogniGron Center, University of Groningen, 9747 AG Groningen, The Netherlands. *email: sylvia.matzen@u-psud.fr; b.noheda@rug.nl
} 
(a)

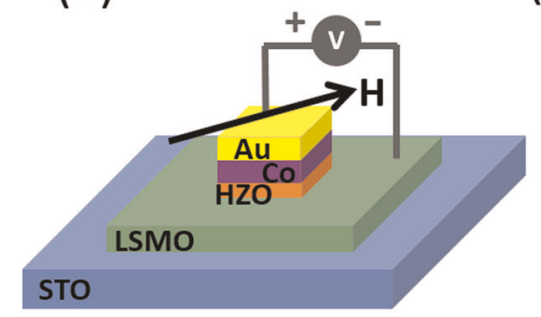

(d)

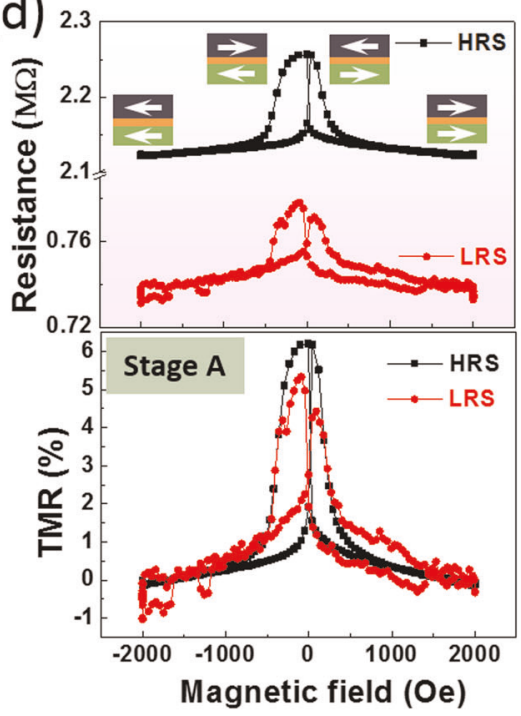

(b) $v$

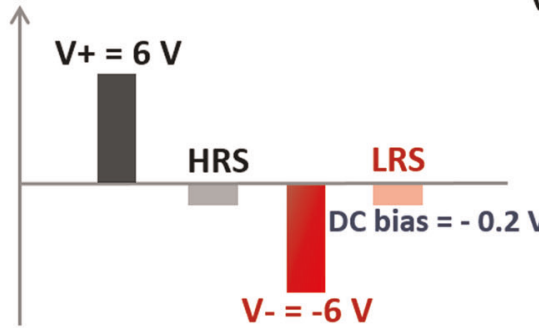

(e)

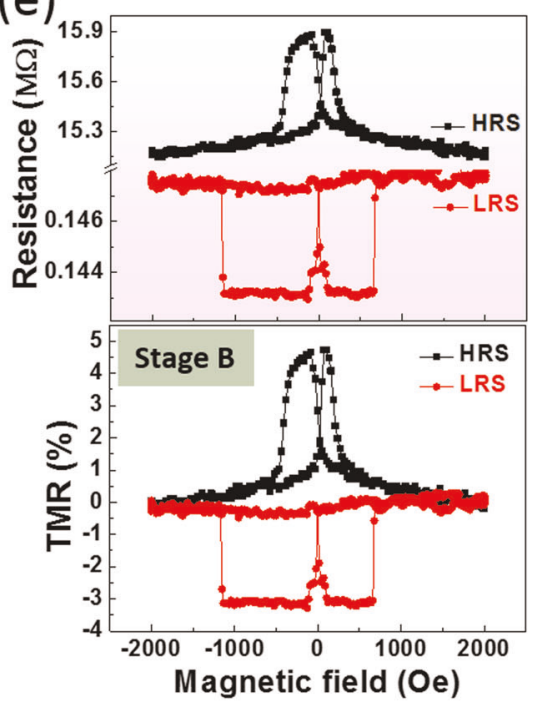

(c)

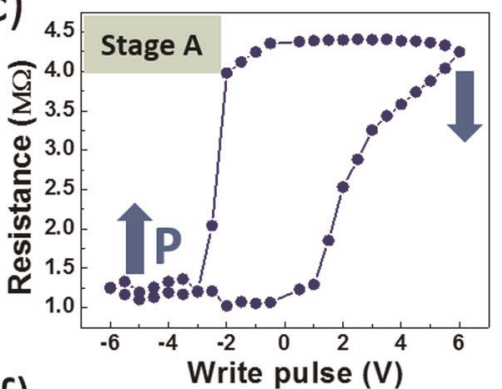

(f)

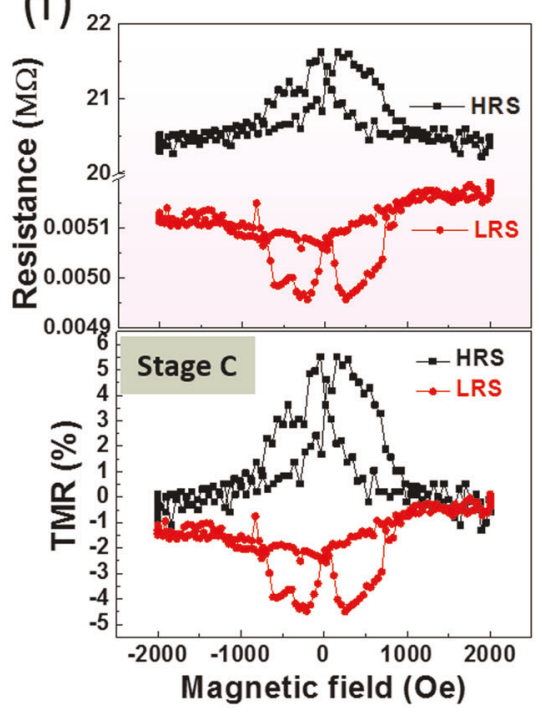

Fig. 1 TMR response under electrical and magnetic stimuli. a Schematic drawing of a tunnel junction device, with bottom electrode grounded. The heterostructure is grown on a 001-oriented $\mathrm{SrTiO}_{3}$ substrate. The applied magnetic field is along the [110] direction. $\mathbf{b}$ Electrical pulses of $6 \mathrm{~V}$ with both positive (black) and negative (red) polarity and $500 \mu$ s duration are applied to the junctions in order to bring them into the high (HRS) and low resistance state (LRS), respectively. All TMR loops are measured under a DC bias of $-0.2 \mathrm{~V}$, both in the HRS and LRS. c Changes in resistance under the application of different amplitude of electrical pulses in the same junction, as shown in Fig. 3a of ref. ${ }^{31}$. d, e, f Resistance as a function of sweeping magnetic field (up panels) and TMR ratio (down panels) in the HRS (black squares) and LRS (red circles) measured at three different intermediate stages (named as stage A, B and C, respectively) upon repeated application of $\pm 6 \mathrm{~V}$ electric pulses. Measurements shown here are performed at $50 \mathrm{~K}$ on a junction device with an electrode area of $30 \times 30 \mu \mathrm{m}^{2}$.

the barrier height that is expected upon the switching of the ferroelectric polarization of the tunnel barrier, ${ }^{1}$ giving rise to a HRS for polarization pointing towards the LSMO layer, and to the LRS for polarization pointing towards the Co layer. It is interesting to notice that LSMO/HZO/Pt junctions, fabricated by Sulzbach et al. ${ }^{32}$ with the same material as tunnel barrier but with a double barrier thickness, also show TER values of around $400 \%$. This TER is reproducible with cycling under relatively smaller driving voltages $(\sim 1 \mathrm{MV} / \mathrm{cm})$, also suggesting that this contribution arises from the ferroelectric polarization.

In both HRS and LRS, TMR loops are obtained, as shown in Fig. $1 \mathrm{~d}$, leading to four resistance states $\left(R_{H_{\uparrow}}, R_{H_{\uparrow \uparrow}}, R_{L_{\uparrow \uparrow}}, R_{L_{\uparrow+}}\right.$, where the arrows signal the relative orientation of the electrodes magnetization). During the first few cycles, the TMR effect of the HRS $(\sim 6.2 \%)$ and LRS $(\sim 5.4 \%)$ are similar in magnitude (see Fig. $1 \mathrm{~d}$ ), indicating a negligible ME coupling, which differs from the strong coupling reported in perovskite tunnel barriers. ${ }^{11,33,34}$ This stage, which we name stage $A$, is the one reported in ref. ${ }^{31}$. Interestingly, after a few tens of cycles, the behaviour changes substantially, reaching the stage $B$, as shown in Fig. 1e: the TMR sign is reversed from positive (HRS) to negative (LRS) indicating that the spin polarization is switched by the external electric field in a reversible manner, as shown in Fig. S1 (see supplementary information). In addition, the coercive field of the harder ferromagnet (the Co layer) in the LRS (with negative TMR) increases by, approximately, a factor of two, compared to the switching fields of the HRS (with positive TMR). Moreover, the switching becomes sharper in the LRS. The increase of the coercive field and steep switching of the Co layer upon electrical cycling could originate from a modification of the HZO/Co interface. ${ }^{24}$ The number of cycles needed to reach the stage $B$ has been found to differ depending on the junction under investigation (for the specific device shown in Fig. 1 , the junction at stage $A / B / C$ is measured after $\sim 20 / 75 / 110$ cycles, respectively).

With further electric cycling (stage C), the TMR signal becomes more noisy, as observed in Fig. If. The switching magnetic fields for the direct and reversed TMR become comparable but still higher than those of stage A (Fig. 1d). However, the magnitude of the TMR effect is not substantially altered. In the meantime, the two magnetic states are less well defined with less abrupt magnetic switching than the previous two stages, which could indicate an increasing number of defects introduced in the stack. For longer cycling time, with number of cycles depending on the junction, the TMR effect eventually disappears but the TER effect is still present.

Loss of bias-induced TMR sign change

Focusing on stage $C$, from the $I-V$ curves measured in parallel and anti-parallel magnetic states, we plot the bias dependence of the TMR for both HRS and LRS in Fig. 2. A striking feature is 
that in HRS state the TMR exhibits a very weak bias-dependence and it is always positive; while in the LRS, the TMR is always negative with a rapid drop of TMR with increasing bias (absolute value), characteristic of thin-film MFTJs and attributed to spin-flip scattering. ${ }^{35}$ The electric field switching of spin polarization is, thus, evidenced over the whole investigated voltage range. Tuning of the read voltage allows to select the magnitude of the TMR change (e.g. Fig. If for $-0.2 \mathrm{~V}$ read voltage). Looking at the bias-dependence of the TMR in the as-grown state for the same device (Fig. 2, inset), and noting that similar curves are obtained in both HRS and LRS at stage A for different junctions, ${ }^{31}$ it is clear that electric field cycling completely changes the control of the spin polarization of the tunneling electrons. While the initial stage A shows a read voltage-controlled TMR sign change, already reported for Co-based junctions, ${ }^{31,36,37}$ in stage $C$ the TMR sign is wholly determined by the switchable resistance state of the device.

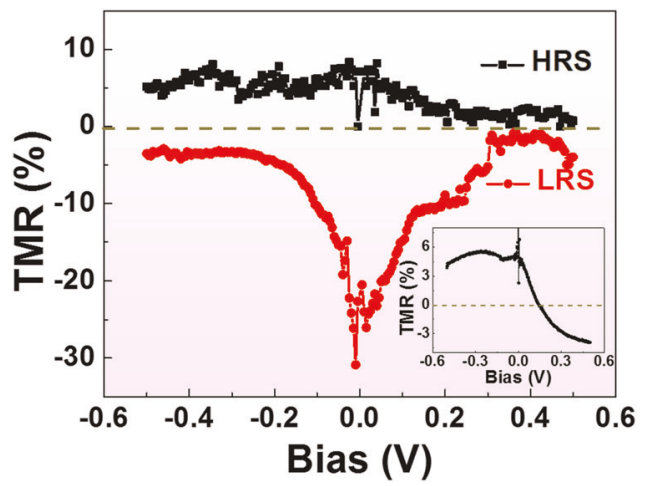

Fig. 2 Bias dependence of TMR measured at stage $C$ for the HRS and LRS, showing electrical switching of spin polarization, on the device shown in Fig. 1f, with size of $30 \mu \mathrm{m} \times 30 \mu \mathrm{m}$, measured at $50 \mathrm{~K}$. The inset shows the bias dependence of TMR in the as-grown state (before any electrical cycling) on the same device. Similar curves are obtained in both HRS and LRS at stage A for a $20 \mu m \times 20 \mu m$ junction, as shown in ref. ${ }^{31}$.
TER built-up

Concomitantly, the resistance ratio between the HRS and LRS (TER) also increases substantially upon electric field cycling. By measuring the current-voltage $(I-V)$ curves after positive and negative electric pulses, we can extract the TER at different bias by measuring the current ratio of HRS and LRS $\left(I_{L} / I_{H}\right)$. TER rises from $10^{2} \%$ to $10^{6} \%$ (stage $A$ to $C$ ) with a large number of intermediate states, as shown in Fig. 3a-c, corresponding to Fig. 1d-f, respectively.

Thus, it is shown that the junctions are strongly affected by the very large electric fields applied across the ultrathin $\mathrm{HZO}$ barrier, which induce stage $B$ and $C$ with highly enhanced magnetoelectric coupling and very large TER, being both features of great interest for devices. The driving voltages required to achieve these stages are close to the junction breakdown field. Therefore, the ability to keep cycling the device with such a large stimulus could be due to the ocurrence of a voltage drop somewhere in the device, such as at the Co-HZO interface. Understanding the mechanisms leading to this evolution would crucially help finding the optimal conditions required for applications in memory devices.

\section{DISCUSSION}

Ion exchange mechanism

To shed light into the factors affecting the evolution from stage A to $C$ by electric cycling, transport measurements of resistance versus temperature $(R-T)$ are shown in Fig. 4a. The same junction is measured in the HRS in stage A (green) and stage C (black). In stage $A$, a metal-insulator transition happens at around $250 \mathrm{~K}$. This is the temperature at which the ferromagnetic/metal-to-paramagnetic/insulator transition of LSMO at the interface with HZO takes place and, thus, where the TMR disappears. ${ }^{31}$ Upon electric field cycling, the transition temperature decreases. In Fig. 4a, the resistance of stage $C$ (black) is shown to display the transition at around $100 \mathrm{~K}$, which again coincides with the temperature at which TMR disappears (see Supplementary Fig. S2). The decrease of transition temperature from stage $A$ to $C$ is consistent with an oxygen deficiency at the LSMO interface ${ }^{38-40}$ that increases with repeated electric field cycling. In addition, the junction $R_{H}$ increases from stage $A$ to $C$ (see Fig. 1), which also agrees with (a)

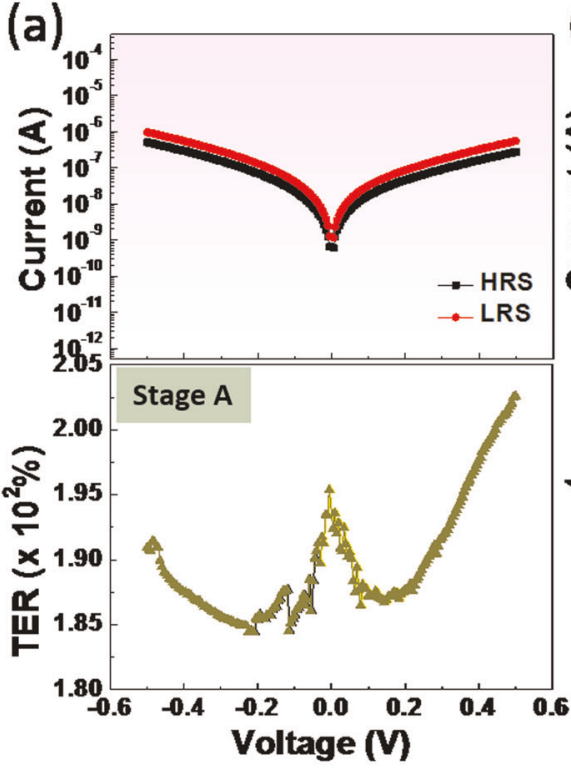

(b)

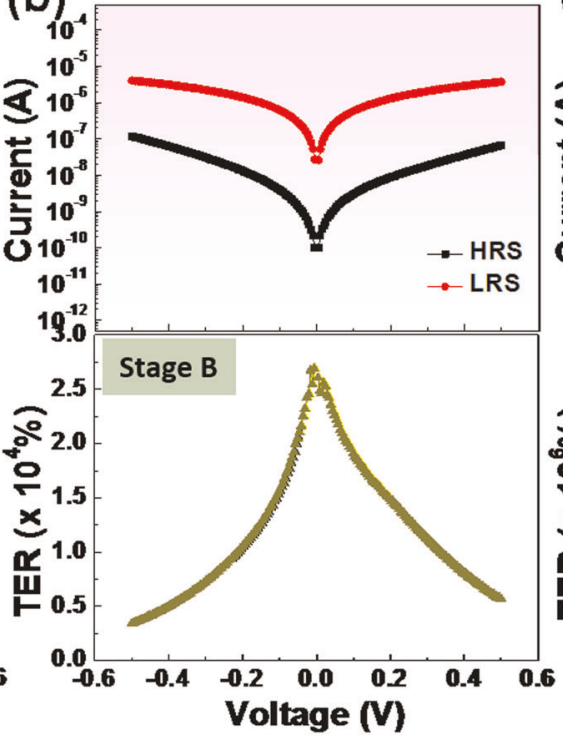

(c)

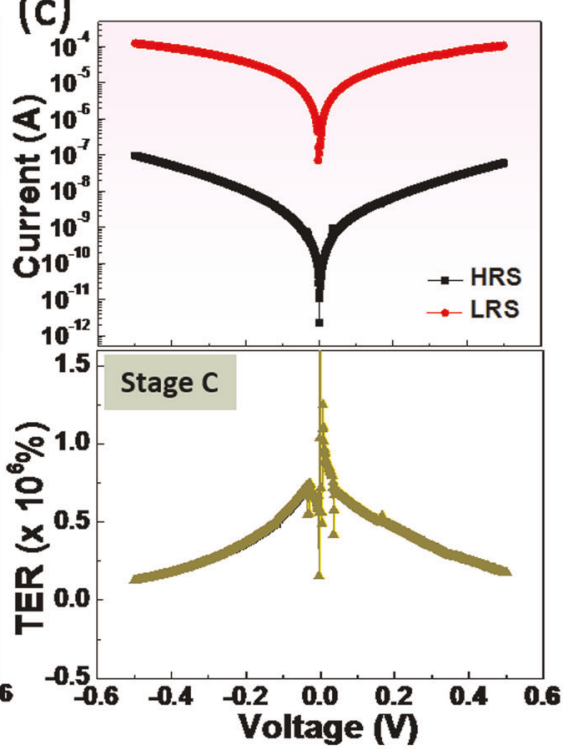

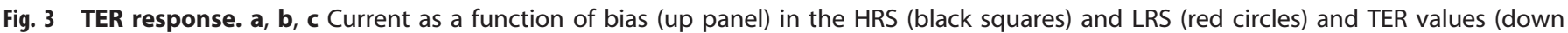
panel) at three different stages $A, B$, and $C$, which correspond to Fig. $1 \mathrm{~d}-\mathrm{f}$. All are measured on a $30 \mu \mathrm{m} \times 30 \mu \mathrm{m}$ junction at $50 \mathrm{~K}$. 


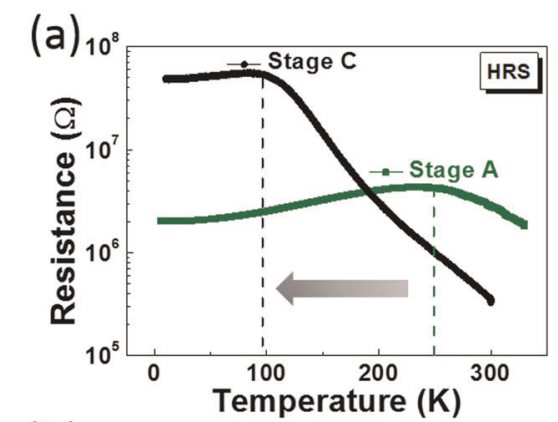

(c)

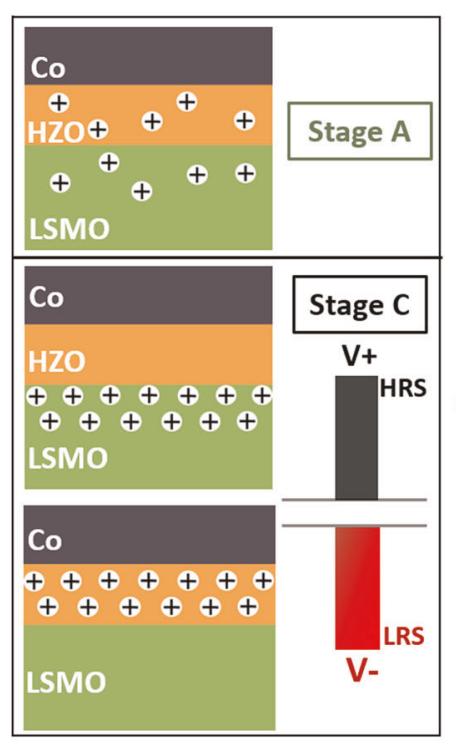

(d)
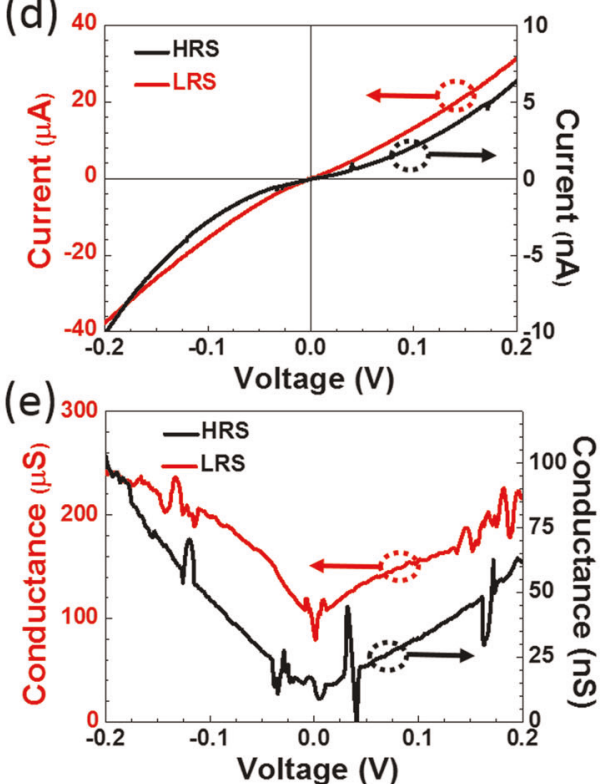

Fig. 4 Ion exchange in a LSMO/HZO/Co stack. a $R-T$ curves in the HRS at stage A (green) and stage $C$ (black), respectively. $\mathbf{b} R-T$ curves in the HRS (black) and LRS (red) at the stage C. c Sketch of the proposed model of interface ionic exchange. d, e $I-V$ and $d l / d V-V$ curves, respectively, in the HRS and LRS of stage $C$ measured at $50 \mathrm{~K}$. The spikes observed in the $d l / d V$ curves are a consequence of the small experimental deviations in the experimental $I-V$ data. Notice the different current/conductance scales in the LRS (left axis) and HRS (right axis).

an increasing content of oxygen vacancies in the LSMO layer at the HRS upon cycling, since oxygen vacancies are well known to reduce the carrier (hole) concentration in LSMO. ${ }^{38,39,41,42}$

Furthermore, the $R-T$ measurements at stage $C$ (with large TER and strong ME coupling) in the HRS and LRS are shown in Fig. 4b. The transition temperature at the HRS (black), which had been lowered by the action of electric cycling to $\sim 100 \mathrm{~K}$, increases up to $\sim 275 \mathrm{~K}$, after the junction is brought to the LRS (red), which is higher than the transition temperature of the stage $\mathrm{A}(\sim 250 \mathrm{~K}$, see Fig. 4a). This indicates that by applying a large negative pulse to the junction, the LSMO layer can reach an oxygen content larger than that of the initial stage. This is consistent with ionic exchange of oxygen vacancies in between the LSMO electrode and the HZO barrier during cycling, as represented in Fig. 4c. Giant resistive switching by oxygen vacancies migration has also been observed in different ferroelectric oxides tunnel barriers. ${ }^{43,44}$

\section{Large TER}

In Fig. 4c, we illustrate this possible scenario: in the as-grown state, both the LSMO and the $\mathrm{HZO}$ layers contain oxygen vacancies $\left(\mathrm{V}_{O}^{2+}\right)$ (top panel). Upon electric field cycling, $\mathrm{V}_{O}^{2+}$ are driven back and forth across the barrier. The evolution of the TER from $10^{2} \%$ to $10^{6} \%$ can be explained by the accumulation of the oxygen vacancies at the vicinity of the HZO/LSMO interface, thus increasing the $\mathrm{V}_{O}^{2+}$ concentration that participates in the ionic exchange process. In this picture, the HRS is due to the oxygen vacancies being pushed into the LSMO electrode, resulting in a very resistive $\mathrm{HZO} / \mathrm{La}_{0.7} \mathrm{Sr}_{0.3} \mathrm{MnO}_{3-\delta}$ contact. The LRS is obtained with the oxygen vacancies drifting back into the HZO barrier upon negative voltage pulse application, greatly reducing the resistivity of the junction. ${ }^{45}$ This gives rise to highly different current levels between HRS and LRS (large TER), as shown in Figs. 3c and 4d. Still, for both states, the non-linear I-V curves are similar (Fig. $4 \mathrm{~d}$ ) and the shape of the differential conductance curves (Fig. 4e) is compatible with tunneling conduction, ${ }^{46}$ ruling out a drastic change of the conduction mechanism as probed by the investigated range of applied voltage.
An open question is the role of the ferroelectric polarization switching in these devices. Resistive switching by electric field has been reported in a wide variety of oxides, ${ }^{45,47,48}$ including binary oxides. ${ }^{49-51}$ In the case of ferroelectric tunnel barriers, the profile of the electronic barrier can be modified by polarization reversal, thus causing strong TER effect. ${ }^{2,52}$ In this picture, the increase of TER effect upon cycling could indicate a concomitant increase of the ferroelectric polarization. This effect has been often observed in hafnia-based ferroelectrics (wake-up effect). ${ }^{53,54}$ Even though our thicker films (down to $5 \mathrm{~nm}$-thick) have not shown that effect, $^{28}$ we are not able to discard its existence in the present $2 \mathrm{~nm}$ tunnel barriers. However, as mentioned earlier, the HRS in the stage A corresponds to downward polarization in Fig. 1c. ${ }^{31}$ Upon evolving into stage $C$, this downward polarization should induce the migration of the oxygen vacancies towards the Co electrode in order to help to screen the polarization charges. However, we observe the contrary: accumulation of oxygen vacancies at the LSMO electrode in the HRS (Fig. 4c) and, thus, an increase of the ferroelectric polarization upon cycling cannot be the main contribution to the TER build-up. Indeed, due to positively charged oxygen vacancies, the screening ability of the LSMO is expected to decrease, which would increase the depolarizing field and strongly reduce the polarization of the tunnel barrier.

\section{ME coupling}

As discussed above, accompanied by the large TER, a strong ME coupling appears with TMR sign reversal. The reversed TMR has been reported to be due to modification of the Co/tunnel barrier interface, such as by adding an interfacial layer, ${ }^{55,56}$ or by electric field control, ${ }^{33}$ similar to our present work. Several microscopic mechanisms have been proposed to explain this phenomenon: (a) by hybridization, the magnetic moment of the interfacial ions can be changed strongly by electrical switching; ${ }^{57}$ (b) the electrostatic contribution at the interface can induce a change of spin polarization in the ferromagnetic layer, ${ }^{58,59}$ and (c) electrochemistry (redox of (Co) at the interface could also cause a change of magnetization. ${ }^{60}$ On the other hand, as shown in Fig. $4 b$ and c, at 
the HRS (stage C), the LSMO/HZO interface is modified, but the TMR remains the same as at the HRS in stage A (Fig. 1d and f). Oxygen vacancies at the LSMO/HZO interface, such as described in Fig. 4c, change neither the amplitude nor the sign of the TMR in the HRS, going from stage A to stage $\mathrm{C}$. We, thus, believe that oxygen migration around this LSMO/HZO interface does not play the main role on the TMR sign reversal. Besides changes at the interfaces, a change of localized (defects) states in the barrier can also induce the inversion of TMR sign due to resonant tunneling. ${ }^{37,61}$ For example, in LSMO/STO/Co junctions, ${ }^{40}$ changes in the barrier by the introduction of $\mathrm{V}_{O}^{2+}$ have been reported to promote TMR sign reversal. In the stage $C$ of our junction, such a migration of $\mathrm{V}_{O}^{2+}$ in the $\mathrm{HZO}$ barrier occurs, back and forth under the application of the high electric field pulses with opposite polarities. Therefore, both the modification of the Co/barrier interface $^{24}$ and the resonant tunneling, could contribute to reversal of the TMR sign.

In conclusion, TER values of up to $10^{6} \%$ coexisting with large ME coupling, by which the sign of the TMR effect is reversed with the electric field switching, have been achieved after cycling of LSMO/ $\mathrm{HZO} / \mathrm{Co}$ tunnel junctions with large enough electric fields. These phenomena can be ascribed to the magneto-ionic effect. The temperature dependence of the transport behaviour is consistent with the exchange of oxygen vacancies at the $\mathrm{LSMO} / \mathrm{HZO}$ interface, together with possible modifications of the $\mathrm{HZO} / \mathrm{Co}$ interface and change of impurity states of tunnel barrier. Next, an electrical protocol needs to be designed in order to increase the endurance of this state.

\section{METHODS}

Thin films of $\mathrm{HZO}$ with thickness of $2 \mathrm{~nm}$ were grown by pulsed laser deposition (PLD) on FM LSMO-buffered (001)-STO substrates. The thickness of LSMO film is around $30 \mathrm{~nm}$. Details of the growth conditions can be found in ref. ${ }^{28}$. In total $50 \mathrm{~nm}$ FM Cobalt with a protective layer of Au $(50 \mathrm{~nm})$, to preserve Co from oxidation, were deposited by sputtering on top of the HZO layer, to form the LSMO (FM)/HZO(FE)/Co (FM) stacks. Junctions with different sizes, ranging from $10 \mu \mathrm{m} \times 10 \mu \mathrm{m}$ to $30 \mu \mathrm{m} \times 30 \mu \mathrm{m}$, are fabricated (see details in ref. ${ }^{31}$ ). The electrical measurements are performed using a Keithley 237 source measurement unit and a Keithley 4200A-SCS parameter analyzer, and the temperature environment and magnetic field are supplied by a Physical Properties Measurement System (PPMS) by Quantum Design. As shown in the schematic drawing in Fig. 1a, the voltage source is applied on the LSMO/ $\mathrm{HZO} / \mathrm{Co}$ stack with bottom electrode grounded (for a positive bias, the electrons are tunneling from LSMO to Co). The magnetic field is swept along the easy magnetization axis of LSMO in the [110] direction.

\section{DATA AVAILABILITY}

The data that support the findings of this study are available from the corresponding authors upon reasonable request.

Received: 25 August 2019; Accepted: 25 November 2019; Published online: 17 December 2019

\section{REFERENCES}

1. Gajek, M. et al. Tunnel junctions with multiferroic barriers. Nat. Mater. 6, 296-302 (2007).

2. Rodríguez Contreras, J. et al. Resistive switching in metal-ferroelectric-metal junctions. Appl. Phys. Lett. 83, 4595 (2003).

3. Tsymbal, E. Y., Gruverman, A., Garcia, V., Bibes, M. \& Barthélémy, A. Ferroelectric and multiferroic tunnel junctions. MRS bull. 37, 138-143 (2012).

4. Scott, J. Multiferroic memories. Nat. Mater. 6, 256-257 (2007).

5. Hur, N. et al. Electric polarization reversal and memory in a multiferroic material induced by magnetic fields. Nature 429, 392-395 (2004).

6. Bibes, M. \& Barthélémy, A. Towards a magnetoelectric memory. Nat. Mater. 7, 425-426 (2008)
7. Ortega, N., Kumar, A., Scott, J. \& Katiyar, R. S. Multifunctional magnetoelectric materials for device applications. J. Phys: Condens. Matter 27, 504002 (2015)

8. Eerenstein, W., Mathur, N. \& Scott, J. F. Multiferroic and magnetoelectric materials. Nature 442, 759-765 (2006).

9. Ramesh, R. \& Spaldin, N. A. Multiferroics: progress and prospects in thin films. Nat. Mater. 6, 21-29 (2007).

10. Fiebig, M. Revival of the magnetoelectric effect. J. Phys. D: Appl. Phys. 38, R123 (2005).

11. Garcia, V. et al. Ferroelectric control of spin polarization. Science 327, 1106-1110 (2010).

12. Yin, Y. \& Li, Q. A review on all-perovskite multiferroic tunnel junctions. J Materiomics 3, 245-254 (2017).

13. Vaz, C. A., Hoffman, J., Ahn, C. H. \& Ramesh, R. Magnetoelectric coupling effects in multiferroic complex oxide composite structures. Adv. Mater. 22, 2900-2918 (2010).

14. Lee, J. H. et al. A strong ferroelectric ferromagnet created by means of spin-lattice coupling. Nature 466, 954-958 (2010).

15. Wang, K., Liu, J.-M. \& Ren, Z. Multiferroicity: the coupling between magnetic and polarization orders. Adv. Phys. 58, 321-448 (2009).

16. Eerenstein, W., Wiora, M., Prieto, J., Scott, J. \& Mathur, N. Giant sharp and persistent converse magnetoelectric effects in multiferroic epitaxial heterostructures. Nat. Mater. 6, 348-351 (2007)

17. Rondinelli, J. M., Stengel, M. \& Spaldin, N. A. Carrier-mediated magnetoelectricity in complex oxide heterostructures. Nat. Nanotechnol. 3, 46-50 (2008).

18. $\mathrm{Lu}, \mathrm{H}$. et al. Electric modulation of magnetization at the $\mathrm{BaTiO}_{3} / \mathrm{La}_{3} \mathrm{Sr}_{3} \mathrm{MnO}_{3}$ interfaces. Appl. Phys. Lett. 100, 232904 (2012).

19. $\mathrm{Vaz}, \mathrm{C}$. et al. Temperature dependence of the magnetoelectric effect in $\mathrm{Pb}\left(\mathrm{Zr}_{0.2} \mathrm{Ti}_{0.2}\right)$ $\mathrm{O}_{0.2} / \mathrm{La}_{0.2} \mathrm{Sr}_{0.2} \mathrm{MnO}_{0.2}$ multiferroic heterostructures. Appl. Phys. Lett. 97, 042506 (2010).

20. Dawber, M., Rabe, K. \& Scott, J. Physics of thin-film ferroelectric oxides. Rev. Mod. Phys. 77, 1083 (2005).

21. Mathews, S., Ramesh, R., Venkatesan, T. \& Benedetto, J. Ferroelectric field effect transistor based on epitaxial perovskite heterostructures. Science 276, 238-240 (1997).

22. Wu, S. et al. Full electric control of exchange bias. Phys. Rev. Lett. 110, 067202 (2013).

23. Skumryev, V. et al. Magnetization reversal by electric-field decoupling of magnetic and ferroelectric domain walls in multiferroic-based heterostructures. Phys. Rev. Lett. 106, 057206 (2011).

24. Bauer, U. et al. Magneto-ionic control of interfacial magnetism. Nat. Mater. 14 174-181 (2015).

25. Böscke, T., Müller, J., Bräuhaus, D., Schröder, U. \& Böttger, U. Ferroelectricity in hafnium oxide thin films. Appl. Phys. Lett. 99, 102903 (2011).

26. Park, M. H. et al. Ferroelectricity and antiferroelectricity of doped thin $\mathrm{HfO}_{2}$ -based films. Adv. Mater. 27, 1811-1831 (2015).

27. Shimizu, T. et al. The demonstration of significant ferroelectricity in epitaxial Ydoped $\mathrm{HfO}_{2}$ film. Sci. Rep. 6, 32931 (2016).

28. Wei, Y. et al. A rhombohedral ferroelectric phase in epitaxially strained $\mathrm{Hf}_{0.5} \mathrm{Zr}_{0.5} \mathrm{O}$ 0.5 thin films. Nat. Mater. 17, 1095-1100 (2018).

29. Lyu, J., Fina, I., Solanas, R., Fontcuberta, J. \& Sánchez, F. Robust ferroelectricity in epitaxial $\mathrm{Hf}_{1 / 2} \mathrm{Zr}_{1 / 2} \mathrm{O}_{1 / 2}$ thin films. Appl. Phys. Lett. 113, 082902 (2018).

30. Lyu, J., Fina, I., Solanas, R., Fontcuberta, J. \& Sánchez, F. Growth window of ferroelectric epitaxial $\mathrm{Hf}_{0.5} \mathrm{Zr}_{0.5} \mathrm{O}_{0.5}$ thin films. ACS Appl. Electron. Mater. 1, 220-228 (2019).

31. Wei, Y. et al. Magnetic tunnel junctions based on ferroelectric $\mathrm{Hf}_{0.5} \mathrm{Zr}_{0.5} \mathrm{O}_{0.5}$ tunnel barriers. Phys. Rev. Appl. 12, 031001 (2019).

32. Sulzbach, M. C. et al. Unraveling ferroelectric polarization and ionic contributions to electroresistance in epitaxial $\mathrm{Hf}_{0.5} \mathrm{Zr}_{0.5} \mathrm{O}_{2}$ tunnel junctions. Adv. Electron. Mater. 1900852 (2019).

33. Pantel, D., Goetze, S., Hesse, D. \& Alexe, M. Reversible electrical switching of spin polarization in multiferroic tunnel junctions. Nat. Mater. 11, 289-293 (2012).

34. Yin, Y. et al. Enhanced tunnelling electroresistance effect due to a ferroelectrically induced phase transition at a magnetic complex oxide interface. Nat. Mater. 12, 397-402 (2013).

35. Moodera, J. S., Nassar, J. \& Mathon, G. Spin-tunneling in ferromagnetic junctions. Annu. Rev. Mater. Sci. 29, 381-432 (1999).

36. De Teresa, J. et al. Inverse tunnel magnetoresistance in $\mathrm{Co} / \mathrm{SrTiO}_{3} / \mathrm{La}_{3} \mathrm{Sr}_{3} \mathrm{MnO}_{3}$ : new ideas on spin-polarized tunneling. Phys. Rev. Lett. 82, 4288 (1999).

37. Tsymbal, E. Y., Sokolov, A., Sabirianov, I. \& Doudin, B. Resonant inversion of tunneling magnetoresistance. Phys. Rev. Lett. 90, 186602 (2003).

38. Cauro, R. et al. Persistent and transient photoconductivity in oxygen-deficient $\mathrm{La}_{2 / 3} \mathrm{Sr}_{2 / 3} \mathrm{MnO}_{2 / 3}$ thin films. Phys. Rev. B 63, 174423 (2001). 
39. Ge, C. et al. Metal-insulator transition induced by oxygen vacancies from electrochemical reaction in ionic liquid-gated manganite films. Adv. Mater. Interfaces 2, 1500407 (2015)

40. Marún, I. V., Postma, F., Lodder, J. \& Jansen, R. Tunneling magnetoresistance with positive and negative sign in $\mathrm{La}_{0.67} \mathrm{Sr}_{0.67} \mathrm{MnO}_{0.67} / \mathrm{SrTiO}_{0.67} / \mathrm{Co}$ junctions. Phys. Rev. B 76, 064426 (2007).

41. Schlueter, $C$. et al. Evidence of electronic band redistribution in $\mathrm{La}_{0.65} \mathrm{Sr}_{0.65} \mathrm{MnO}$ 0.65 by hard x-ray photoelectron spectroscopy. Phys. Rev. B 86, 155102 (2012).

42. Yao, L., Inkinen, S. \& Van Dijken, S. Direct observation of oxygen vacancy-driven structural and resistive phase transitions in $\mathrm{La}_{2 / 3} \mathrm{Sr}_{2 / 3} \mathrm{MnO}_{2 / 3}$. Nat. Commun. 8, 14544 (2017).

43. Qin, Q. H. et al. Resistive switching in all-oxide ferroelectric tunnel junctions with ionic interfaces. Adv. Mater. 28, 6852-6859 (2016).

44. Kim, D. et al. Ferroelectric tunnel memristor. Nano Lett. 12, 5697-5702 (2012).

45. Waser, R., Dittmann, R., Staikov, G. \& Szot, K. Redox-based resistive switching memories-nanoionic mechanisms, prospects, and challenges. Adv. Mater. 21, 2632-2663 (2009).

46. O'donnell, J., Andrus, A., Oh, S., Colla, E. \& Eckstein, J. Colossal magnetoresistance magnetic tunnel junctions grown by molecular-beam epitaxy. Appl. Phys. Lett. 76, 1914 (2000)

47. Sawa, A. Resistive switching in transition metal oxides. Mater. Today 11, 6 (2008).

48. Waser, R. \& Aono, M. Nanoionics-based resistive switching memories. Nat. Mater. 6, 833-840 (2007).

49. Seo, S. et al. Reproducible resistance switching in polycrystalline NiO films. Appl. Phys. Lett. 85, 5655 (2004).

50. Simmons, J. \& Verderber, R. New conduction and reversible memory phenomena in thin insulating films. Proc. R. Soc. A. 301, 77-102 (1967).

51. Choi, B. et al. Resistive switching mechanism of $\mathrm{TiO}_{2}$ thin films grown by atomiclayer deposition. J. Appl. Phys. 98, 033715 (2005).

52. Gruverman, A. et al. Tunneling electroresistance effect in ferroelectric tunnel junctions at the nanoscale. Nano Lett. 9, 3539-3543 (2009).

53. Hoffmann, M. et al. Stabilizing the ferroelectric phase in doped hafnium oxide. J. Appl. Phys. 118, 072006 (2015).

54. Starschich, S., Menzel, S. \& Böttger, U. Evidence for oxygen vacancies movement during wake-up in ferroelectric hafnium oxide. Appl. Phys. Lett. 108, 032903 (2016).

55. De Teresa, J. M. et al. Role of metal-oxide interface in determining the spin polarization of magnetic tunnel junctions. Science 286, 507-509 (1999).

56. LeClair, P. et al. Sign reversal of spin polarization in $\mathrm{Co} / \mathrm{Ru} / \mathrm{Al}_{2} \mathrm{O}_{2} / \mathrm{Co}$ magnetic tunnel junctions. Phys. Rev. B 64, 100406(R) (2001).

57. Valencia, S. et al. Interface-induced room-temperature multiferroicity in $\mathrm{BaTiO}_{3}$. Nat. Mater. 10, 753-758 (2011).

58. Zhang, S. Spin-dependent surface screening in ferromagnets and magnetic tunnel junctions. Phys. Rev. Lett. 83, 640 (1999).

59. Zhuravlev, M. Y., Maekawa, S. \& Tsymbal, E. Y. Effect of spin-dependent screening on tunneling electroresistance and tunneling magnetoresistance in multiferroic tunnel junctions. Phys. Rev. B 81, 104419 (2010).

60. Bowen, M. et al. Bias-crafted magnetic tunnel junctions with bistable spindependent states. Appl. Phys. Lett. 89, 103517 (2006).

61. Klyukin, K., Tao, L., Tsymbal, E. Y. \& Alexandrov, V. Defect-assisted tunneling electroresistance in ferroelectric tunnel junctions. Phys. Rev. Lett. 121, 056601 (2018).

\section{ACKNOWLEDGEMENTS}

We are indebted to Alexei Gruverman and Pratyush Buragohain for many enlightening conversations. We are also grateful to Tamalika Banerjee and Manuel Bibes for useful discussions and to Jacob Baas and Henk Bonder for their technical support. Y.W. and B.N. acknowledge a China Scholarship Council grant and a Van Gogh travel grant.

\section{AUTHOR CONTRIBUTIONS}

Y.W, S.M., and B.N. devised the concept. Y.W. grew the heterostructures and performed all the characterizations prior and post the fabrication of the devices. S.M., G.A., and T.M. fabricated the devices, with the help of P.L. The electrical response of the devices was analyzed by Y.W., S.M., T. M., G.A., and C.P.Q. All the authors discussed the data. Y.W. and B.N. wrote the manuscript with the feedback and input of S.M., G. A., T.M., and C.P.Q.

\section{COMPETING INTERESTS}

The authors declare no competing interests.

\section{ADDITIONAL INFORMATION}

Supplementary information is available for this paper at https://doi.org/10.1038/ s41535-019-0201-0.

Correspondence and requests for materials should be addressed to S.M. or B.N.

Reprints and permission information is available at http://www.nature.com/ reprints

Publisher's note Springer Nature remains neutral with regard to jurisdictional claims in published maps and institutional affiliations.

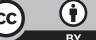

Open Access This article is licensed under a Creative Commons Attribution 4.0 International License, which permits use, sharing, adaptation, distribution and reproduction in any medium or format, as long as you give appropriate credit to the original author(s) and the source, provide a link to the Creative Commons license, and indicate if changes were made. The images or other third party material in this article are included in the article's Creative Commons license, unless indicated otherwise in a credit line to the material. If material is not included in the article's Creative Commons license and your intended use is not permitted by statutory regulation or exceeds the permitted use, you will need to obtain permission directly from the copyright holder. To view a copy of this license, visit http://creativecommons. org/licenses/by/4.0/.

(c) The Author(s) 2019 